\title{
Beta-Lactam Antibiotic
}

National Cancer Institute

\section{Source}

National Cancer Institute. Beta-Lactam Antibiotic. NCI Thesaurus. Code C260.

A compound with a four member amide ring (beta-lactam), where one member is the amide nitrogen and another is the carbonyl carbon, with bactericidal activity. Beta-lactam antibiotics act by disrupting the synthesis of the peptidoglycan layer of the bacterial cell wall. 